\title{
The Improved Capabilities of the Goldstone Solar System Radar Observatory
}

\author{
Nereida Rodriguez-Alvarez ${ }^{\circledR}$, Senior Member, IEEE, Joseph S. Jao, Clement G. Lee, \\ Martin A. Slade, Member, IEEE, Joseph Lazio, Kamal Oudrhiri, Kenneth S. Andrews, \\ Lawrence G. Snedeker, Ronglin R. Liou, and Kevin A. Stanchfield
}

\begin{abstract}
The Goldstone Solar System Radar (GSSR) facility is the largest fully steerable ground-based radar in the world for nonclassified high-resolution ranging and imaging of planetary and small-body targets. Over the years, the use of the GSSR to detect and characterize near-Earth objects (NEOs) has become critical to keep track of potential Earth-impacting hazardous NEO. This article relates the specific modifications made to the GSSR hardware and procedures in the last few years, as well as the new capabilities derived from those upgrades: reduced complexity in remote operations, increased experimental design versatility, and increased performance on bistatic radar experiments from GSSR to other complexes. In addition, we dedicate a section to provide an update on the current GSSR power capabilities as the new klystrons are installed. The work detailed in this article is intended to reach the broader science community in order to communicate how those modifications and the derived new capabilities can make science experiments more successful.
\end{abstract}

Index Terms-Goldstone Solar System Radar (GSSR), planetary radar, observatory.

\section{INTRODUCTION}

$\mathbf{T}$ HE Goldstone Solar System Radar (GSSR) originated in 1958 during the beginning of the National Aeronautics and Space Administration (NASA) space program, as NASA recognized that planetary research required a two-way communication capability on Earth. The GSSR is located in Barstow, California, at one of the three Deep Space Network (DSN) sites, and its technology was developed by the Jet Propulsion Laboratory (JPL), universities, and industry. The requirements on the antenna size and the high-power transmitter were derived from projects such as Project Echo (1960-1964, [1])

Manuscript received January 14, 2021; revised April 20, 2021; accepted April 21, 2021. This work was supported in part by the Jet Propulsion Laboratory, California Institute of Technology, under a contract with the National Aeronautics and Space Administration and in part by the DSN O\&M Project-SSR Tech Leadership 2020, California Institute of Technology. (Corresponding author: Nereida Rodriguez-Alvarez.)

Nereida Rodriguez-Alvarez, Joseph S. Jao, Clement G. Lee, Martin A. Slade, Joseph Lazio, Kamal Oudrhiri, Kenneth S. Andrews, Ronglin R. Liou, and Kevin A. Stanchfield are with the Jet Propulsion Laboratory, California Institute of Technology, Pasadena, CA 91109 USA (e-mail: nereida.rodriguez.alvarez@jpl.nasa.gov; joseph.s.jao@jpl.nasa.gov; clement.g.lee@jpl.nasa.gov; martin.a.slade@jpl.nasa.gov; joseph.lazio@ jpl.nasa.gov; kamal.oudrhiri@jpl.nasa.gov; kenneth.s.andrews@jpl.nasa.gov; ronglin.r.liou@jpl.nasa.gov; kevin.a.stanchfield@jpl.nasa.gov).

Lawrence G. Snedeker is with Peraton Inc., Monrovia, CA 91016 USA, and also with the Jet Propulsion Laboratory, California Institute of Technology, Pasadena, CA 91109 USA (e-mail: lawrence.g.snedeker@jpl.nasa.gov).

Digital Object Identifier 10.1109/TGRS.2021.3084861 at the DSN, which in turn benefited the GSSR technology developments. The relevance of the GSSR grew with the passing of the years as the science community soon realized its potential to obtain critical information to design spacecraft missions to planets, asteroids, moons, and comets. From its creation, the GSSR has supported observations of Venus (see [2]-[4]), Mercury (see [5]-[7]), Moon (see [8], [9]), Mars (see [10]-[12]), the Galilean satellites [13], and Titan (see [14, [15]). More recently, the use of GSSR has been focused primarily in the tracking and characterization of NEOs (see [16]-[25]). As NASA funded more experiments, those experiments helped in defining new requirements to build new capabilities that in turn helped the GSSR to stay at the leading edge of planetary radar technology.

This article describes the modifications and the new capabilities added to the GSSR in the last few years. The developments allow the automation of the majority of GSSR operations of the radar and make possible the definition of new experiments. In order to provide to the reader a complete understanding of the GSSR, in Section II, we include, summarized from [26] and [27], an overview of the GSSR elements, the modes of observation, an overview of the GSSR hardware interface, and the type of measurements that can be carried out. Section III describes the capabilities already existing by describing the different experiments that are regularly conducted and the science that can be accomplished through those experiments. Section IV describes the specific modifications made on the GSSR that have enabled new capabilities: reduced complexity in remote operations, increased experimental design versatility, and increased performance on bistatic radar experiments from GSSR to other observatories. Section V is dedicated to provide an updated status on the GSSR power capabilities, as new klystrons are being delivered by Communications and Power Industries (CPIs) to the Jet Propulsion Laboratory (JPL) and being installed at the Deep Space Station (DSS) at Goldstone DSN, enabling GSSR with improved power capabilities restoring it to full-power operations. A conclusion is provided in Section VI.

\section{GSSR: DESCRIPTION}

This section provides an overview of the GSSR, describing its main elements, different modes of observation, the GSSR's hardware interface, and the measurements that are regularly 


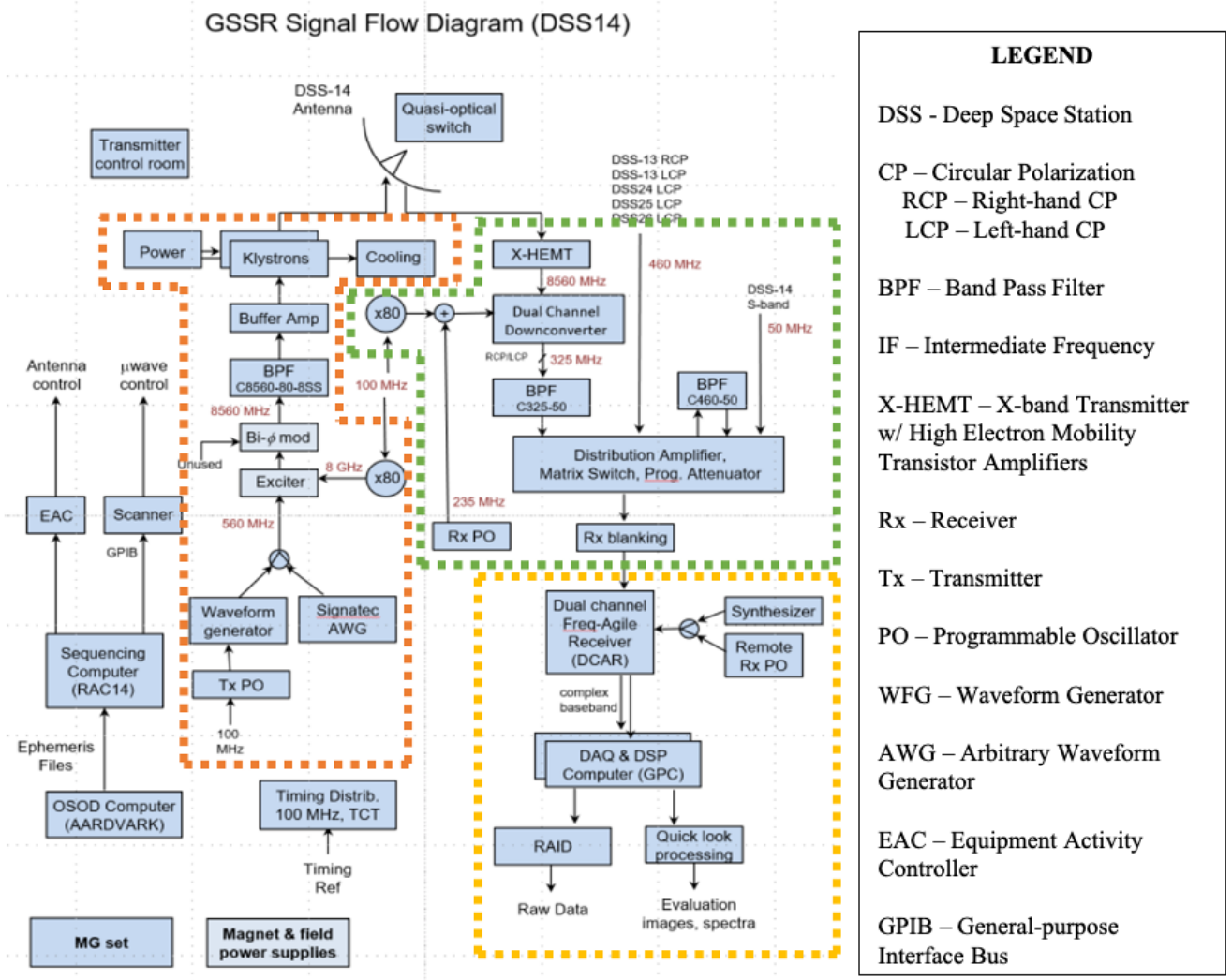

Fig. 1. Detailed block diagram of all GSSR hardware parts. Orange box delimits the transmitter system, green box delimits the receiving system, and yellow box delimits the DAS. A legend is provided on the right side.

carried out. For extended information on this section, refer to [26] and [27].

\section{A. GSSR Elements}

The main elements of the GSSR are the transmitter, the antenna, the receiver, and the data acquisition subsystems (DASS). The GSSR block diagram is shown in Fig. 1.

The GSSR systems have been marked in three colored boxes: the orange dashed box includes the transmitter elements, the green dashed box includes the receiver elements, and the yellow dashed box includes the DAS elements. Also, the antenna elements are shown at the top of Fig. 1: DSS-14 antenna and the quasi-optical switch. All these system elements are further described in Sections II-A1-II-A4.

1) GSSR Transmitter: The GSSR high-power transmitter subsystem operates at the X-band (8560 MHz), [28], and is capable of radiating $\sim 450 \mathrm{~kW}$ by low-loss power combining the output from two $250-\mathrm{kW}$ klystrons. The transmitter signal sources from the transmitter programmable oscillator (Tx PO, Fig. 1), which has an internal 1.12-GHz crystal oscillator, are referenced by the station's $100-\mathrm{MHz}$ clock. The $560-\mathrm{MHz}$ output signal is then modulated with a baseband waveform that is generated from the waveform generator. Alternatively, as it will be described in the new capabilities, an Arbitrary Waveform Generator (Signatec AWG, Fig. 1) has been added to the GSSR systems and allows the generation of more versatile waveforms. This waveform is injected into the signal path via an exciter, mixing it with an $8-\mathrm{GHz}$ signal to produce the desired $8560-\mathrm{MHz}$ waveform, which is then filtered and amplified to condition it for amplification by two klystrons. Klystrons are high-power amplifiers [29], invented in 1937 by American electrical engineers Russell and Sigurd Varian, based on the principle of an electron beam interacting with radio waves as it passes through resonant cavities, metal boxes, along the length of a tube. GSSR klystrons are designed by CPI. The klystrons are design to produce at least $250 \mathrm{~kW}$ of output power at a beam voltage not exceeding $53 \mathrm{kV}$ [29]. Klystrons achieve a bandpass, whose spectrum is nearly flat across the central $45 \mathrm{MHz}$ with a variation of less $<1 \mathrm{~dB}$, and an excellent gain, $>49 \mathrm{~dB}$, which surpasses the requirements for performance [29]. Klystrons are vacuum tube electron beam devices that are the key amplifying elements of most radio frequency telecommunications and radar transmitter systems. The main elements of the GSSR klystrons [29] are the electron gun, the multicavity RF body, the solenoid focusing magnet, the output circuit and vacuum window, and the collector for the spent electron beam. An updated status of the GSSR transmitter current power capabilities is provided in Section V.

The abovementioned waveform generator can provide, therefore, a constant radio frequency signal with no time modulation for the continuous wave $(\mathrm{CW})$ mode, a pseudo-noise (PN) code modulation for the binary phased-coded (BPC) mode, or a linear frequency modulation (LFM) for the chirping mode. Those waveform types are further explained in 


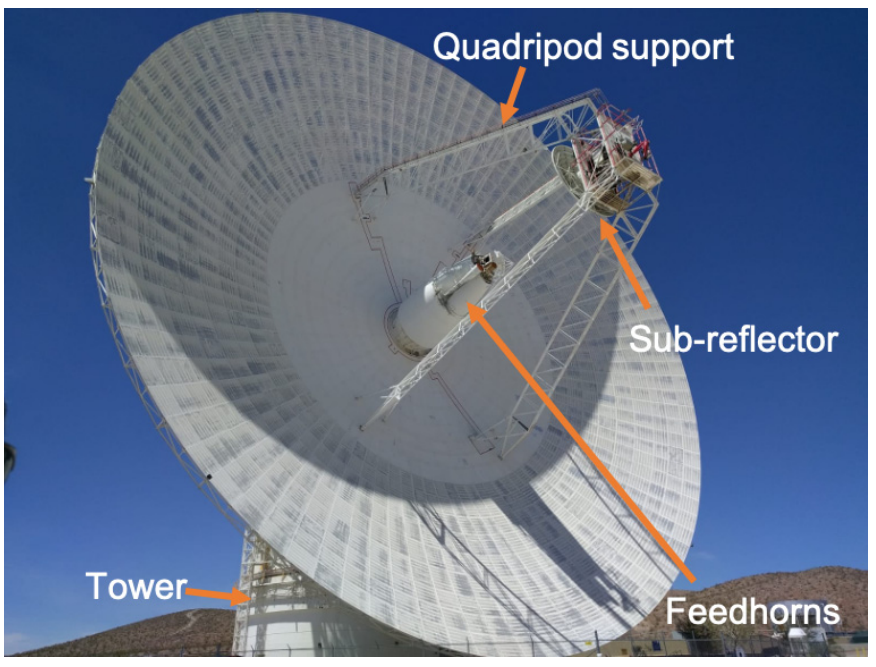

Fig. 2. DSS-14 70-m dish antenna (photograph taken by author on May 4, 2018).

TABLE I

GSSR TRANSMitTer PERFormance

\begin{tabular}{lc}
\hline \hline \multicolumn{1}{c}{ Parameter } & Value \\
\hline Antenna Gain & $73.23 \pm 0.2 \mathrm{dBi}$ \\
Antenna Beamwidth & $0.038 \pm 0.004 \mathrm{deg}$. \\
Polarizations & $\mathrm{RC}, \mathrm{LC}$ \\
Frequency Accuracy & $\pm 3 \times 10^{-13}$ \\
Cross-polarization & $-25 \mathrm{~dB}$ \\
\hline \hline
\end{tabular}

Section III. Table I lists the specifications for the GSSR transmitter performance, which are obtained from [30].

2) GSSR Antenna: The GSSR antenna subsystem is composed of a 70-m-diameter dish (Deep Space Station 14 [DSS-14]), a subreflector, a supporting quadripod, a the pointing system. Fig. 2 shows an illustration of the DSS-14 70-m antenna and its main components.

The 70-m antenna is a fully steerable beam-shaped reflector with horn feeds and Cassegrain configuration [31]. Its surface consists of $\sim 1200$ precision-shaped aluminum trapezoidal panels individually adjusted for optimum performance. The required accuracy is $1 / 16$ of a wavelength, but there are small distortions on the antenna shape as a function of the elevation pointing angle that results in small loss gain due to the varying stress from gravity on the structure and wind loading and thermal stress. The panels near the center of the dish are solid, but the panels away from the center are perforated to reduce weight and the effect of wind. The effect of mechanical surface deviation on antenna gain is $\sim 0.5 \mathrm{~dB}$ at $8560 \mathrm{MHz}$. The subreflector is mounted on a quadripod support inside the main antenna dish (Fig. 2). During radar operations, the subreflector is focused on the transmit horn. The generated signal is sent by the transmitter through the klystron amplifiers and out the GSSR transmitter horn, where a quasi-optical transmit/receive switch (QOS, also referred to as
TABLE II

GSSR ANTENNA MAIn CHARACTERISTICS

\begin{tabular}{lc}
\hline \hline \multicolumn{1}{c}{ Parameter } & Value \\
\hline Elevation Coverage & {$[10,89.5] \mathrm{deg}$.} \\
Azimuth Coverage & Full \\
Pointing Accuracy & $0.002 \mathrm{deg}$. \\
Effective Collecting Area & $2.5 \times 10^{3} \mathrm{~m}^{2}$ \\
Aperture Efficiency & $64 \%$ \\
\hline \hline
\end{tabular}

a mirror) is used to provide rapid switching from transmission to receiving and vice versa. The QOS moves over the transmit horn during receiving to direct the RF energy to the receive horn. The pointing system is stable, as it is installed in the rotatable tower-a vibration-free platform independent from the pedestal-and allows for remote pointing control carried out from the signal processing center-house in an adjacent building. The pointing allows target detection of the entire sky north of declination $-50^{\circ}$ and can achieve nearly $11 \mathrm{~h}$ of tracking at $35^{\circ}$ declination. The high-accuracy pointing is obtained by implementing a master equatorial pointing method [30]. Table II summarizes the GSSR antenna main characteristics.

3) GSSR Receiver: After the signal arrives to the antenna, the receiver amplifies the echoes received by the antenna, which are extremely weak, through a high-electron mobility transistor (HEMT) low-noise amplifier (LNA) (see Fig. 1). To optimize the amplifier chain, the front-end receiver stages are located at a minimum distance from the feedhorns in the antenna. The rest of the receiver stages occurs in the pedestal of the antenna. In addition, the receiver processes two identical receiver channels, using two HEMT-LNA and providing two orthogonal circular polarizations (left- $L C$ and right- $R C$ ), commonly referred to as same sense (SC) and opposite sense (OC), depending on the transmitter polarization.

Next, the signal goes into the downconversion stage. The downconversion follows a superheterodyne scheme, where the radio signal is converted to lower frequencies in various steps, intermediate frequencies (IFs), by mixing the radar echoes with frequency stable pure sine waves or local oscillators (LOs). The downconverter, therefore, mixes the RF produced from the HEMT-LNAs with the LO (RX LO, Fig. 1) signal to produce an IF centered at $325 \mathrm{MHz}$. The receiver then outputs the signal to the DAS. Table III shows a summary of the GSSR receiver performance, whose values are obtained from [30].

4) GSSR DAS: The DAS is critical for conversion of the analog signals from the receiver-to-digital data (A/D converters) that is recorded to disks for offline data analysis. Fig. 1 shows the DCAR, a back-end receiver that digitizes the abovementioned IF output, and a signal of 50-MHz bandwidth centered at $325 \mathrm{MHz}$.

The software that is used to command and control the radar is included in the DAS. Parts of that software use ephemerides information to drive the POs, an important part of the GSSR 
TABLE III

GSSR RECEIVER PERFormANCE

\begin{tabular}{lc}
\hline \hline \multicolumn{1}{c}{ Parameter } & Value \\
\hline Antenna Gain & $74.55 \pm 0.1 \mathrm{dBi}$ \\
Antenna Beamwidth & $0.032 \pm 0.002 \mathrm{deg}$. \\
Polarizations & $\mathrm{RC}, \mathrm{LC}$ \\
Sensitivity & $0.9 \mathrm{~K} / \mathrm{Jy}$ \\
Pointing Loss & $0.02 \mathrm{deg}$. \\
Cross-polarization & $-25 \mathrm{~dB}$ \\
\hline \hline
\end{tabular}

system. The data acquisition and digital signal processing (DAQ-DSP, Fig. 1) computer carries out the appropriate signal processing depending on the goals of the particular observation.

1) For CW signals, the DAS computes the power spectrum of the received signal.

2) For BPC and LFM signals, data reduction is also carried out by the DAS by convolving the received signal with a replica of the transmitted signal. The FFT is applied to produce a delay-Doppler image.

\section{B. Mode of Observation}

In this section, the two primary modes of operation are summarized: monostatic and bistatic. A multistatic (interferometry) implementation is also possible when more than two receive antennas are involved in the measurements.

1) Monostatic Observation: Monostatic radar involves a single antenna. At Goldstone, only DSS-14 is currently capable of carrying out such an observation. The QOS, explained in Section II-A2, installed on top of X-band and K-band receiver (XKR) cones, allows the radar waveform generated by the transmitter to exit the transmitting horn illuminating the antenna, and allows the antenna to illuminate the receiving horn, so the DSS-14 can rapidly switch from a transmit position to a receive position, and vice versa.

At the start of a monostatic run, the QOS is configured for transmission and an interlock switch is engaged to protect the LNA from any scattered radiation entering the receive horn, i.e., the safety of the transmitter is preserved with proper interlock protocol. Transmissions occur for the full interval of the round-trip (RT) light travel time to the target. Then, the quasi-optical switch is configured for reception, while the interlock switch is disengaged. The returning radar reflection is recorded before the second RT time ends, and then data reduction starts. This procedure is usually repeated multiple times. The minimum distance at which monostatic observations can be conducted is 4 light seconds because the quasi-optical switch and interlock switch cannot be completed faster than $3 \mathrm{~s}$.

2) Bistatic Observation: A bistatic track involves two antennas, one transmits and one receives operating with overlapped bandwidths. The GSSR signal can be received by other
TABLE IV

List OF BISTATIC RADAR OPERATION OPPORTUNITIES USING THE Signal TRANSMitTER BY THE GSSR. NOTE THAT AO IS No LONGER OPERATIONAL

\begin{tabular}{|c|c|c|c|c|}
\hline \multirow[b]{2}{*}{$\begin{array}{c}\text { DSS14 } \\
\text { Transmitter } \\
\text { Configuration }\end{array}$} & \multirow[b]{2}{*}{$\begin{array}{c}\text { Agency } \\
\text { Type }\end{array}$} & \multirow[b]{2}{*}{$\begin{array}{l}\text { Receiving } \\
\text { Antenna }\end{array}$} & \multicolumn{2}{|c|}{ Receiver } \\
\hline & & & $\begin{array}{c}\text { Frequency } \\
\text { Range } \\
(\mathrm{MHz})\end{array}$ & $\begin{array}{l}\text { Tsys } \\
(\mathrm{K})\end{array}$ \\
\hline \multirow{6}{*}{$\begin{array}{c}\text { X-Band, } \\
450 \mathrm{~kW}, \\
{[8535-8585]} \\
\mathrm{MHz}\end{array}$} & Inter & GBT & $8000-10100$ & 27 \\
\hline & Inter & $\mathrm{AO}$ & $8000-10000$ & 32 \\
\hline & Intra & DSS-13 & $6800-9200$ & 15 \\
\hline & Intra & DSS-28 & $4000-14000$ & 44 \\
\hline & Intra & DSS-25 & $8400-8600$ & 22 \\
\hline & Intra & DSS-26 & $8400-8600$ & 22 \\
\hline
\end{tabular}

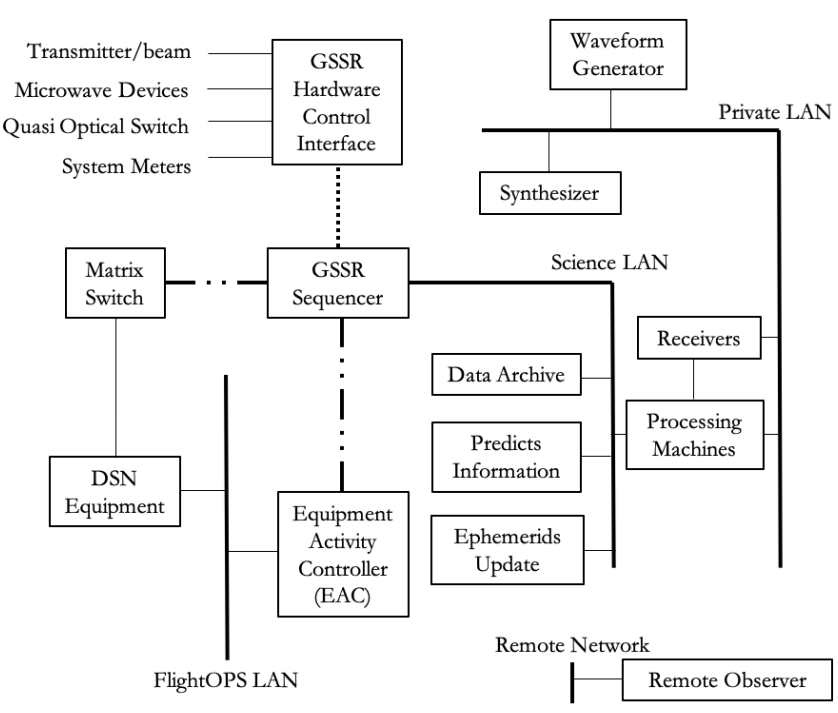

Fig. 3. General connection diagram with all GSSR links to other subsystems.

antennas to carry out bistatic observations. These antennas can be part of the DSN, intraagency observations, or antennas located at other complexes, interagency observations, such as Arecibo Observatory (AO) and Green Bank Telescope (GBT). Table IV summarizes the antennas that can receive the GSSR signal.

As Table IV shows, there are six possible combinations using the GSSR as the transmitter. The advantage of using interagency's antennas for bistatic experiments, such as GBT or in the past $\mathrm{AO}$, is the size of the antennas: the GBT antenna's diameter is $100 \mathrm{~m}$ and the $\mathrm{AO}$ antenna's diameter is $305 \mathrm{~m}$. Larger antennas provide higher receiving gain and, therefore, more sensitivity to the target features.

\section{Overview of the GSSR Hardware Interface}

This section describes the GSSR hardware interface. Fig. 3 shows a high-level connection diagram with all GSSR links to other subsystems in order to provide a general overview of the networks and the elements within each network that are needed for the operation of the GSSR. 
The general connection diagram in Fig. 3 shows the GSSR links to other subsystems through different local area network (LAN) and secure connections. The following specific LAN is involved:

1) Flight operations (FlightOPS) LAN is a highly secured network used for housing DSN equipment, such as antenna pointing controller and subreflector.

2) Science LAN is an internal network used for GSSR computers that store configuration files (e.g., ephemerides and real-time predict files), temporary data storage, and archive data.

3) Private LAN is an internal network used by the processing machines, as well as the support hardware, such as synthesizers, waveform generators, and receivers.

4) Remote network is an external network used for visualization at machines outside the private network, where an observer can follow the postprocessing and the evolution of the experiments. One of the processing machines, inside the private LAN, transfers the processed images into the remote location.

The main brain is the GSSR sequencer which controls the transmitter, microwave devices, QOS, and other system meters, such as power and temperature, as well as communicates with the equipment access controller (EAC) and matrix switch through secure connections.

For transmission, the GSSR sequencer commands the waveform generator that generates the desired waveform, either standard CW, BPC, or chirp, or some more versatile waveform using the new arbitrary waveform generator (described in Section IV). The waveform is then provided to the exciter and it continues through various elements as shown in Fig. 1. The GSSR waveform generator has an option to generate Doppler precompensated waveforms. The advantage of precompensating Doppler is to minimize the receive signal processing time so that the results are available in real time. With that information, the ephemeris can be refined and updated. The ephemeris provides the antenna with the object's trajectory information. Typically, ephemeris files are prepared by the principal investigator (P.I.) and are provided to the GSSR sequencer, which incorporates the information on those files into the system. The pointing information is then sent to the Antenna Pointing Controller Assembly (APCA) via the EAC to ensure the antenna points in the correct direction. The same file is also loaded into waveform generator, if Doppler precompensation is needed, and into the back-end receiver/processing machine for an absolute range calculation. The ephemeris file provides the RT light-time from the transmitter to the target and then back to the receiver. With RT time, the GSSR can calculate Doppler's frequency shift and time delay for range phase.

During reception, once the samples from the receivers arrive to the processing machines, real-time processing can be carried out. The scientists analyze the data and make active decisions, such as changing the waveform, increasing the baud rate, or other parameters, and update ephemerides accordingly. New set-ups are then introduced by the operator into the system through an automated software package, and the GSSR sequencer reads in all the information, as well as the updated ephemeris files. The experiments, therefore, evolve as the track advances and the resolution is dynamically improved.

\section{GSSR Measurements}

The received radar echo is further processed and analyzed in terms of amplitude, time delay, Doppler shift, and polarization-related properties. For extended information on this section, refer to [26] and [27].

1) Amplitude Measurements: The amplitude or strength of the radar echo, received from a target, needs to be compensated by the RT loss at interplanetary distances, transmitted power, and antenna gain. The integration time required to have detection depends on the signal-to-noise ratio (SNR) at the input of the receiver. Some objects can be detected in a few seconds, while some others may take minutes. After compensation and integration, the amplitude signal can derive the radar cross section of the target and a measure of how much of the incident energy reflects back from the target. A 3:1 SNR ratio after processing is usually the minimum required for reliable detectability.

The amplitude, linked to the radar cross section, is a measure of surface roughness and dielectric constant. Bright/strong pulses are usually associated to smooth-or surfaces with the right slope to be reflected back to Earth—and/or highly reflective surfaces. Dark/weak pulses are usually associated to rough surfaces-or smooth surfaces sloped so that the incident wave reflects away from Earth - and/or surfaces very absorptive at the wavelength used. The rougher the surface is the more diffuse is the scattering and the broader the received pulse appears in the frequency domain.

2) Time-Delay Measurements: Precise measurements of the distance between the target and Earth are obtained by measuring the time between the instant that the signal leaves the transmitter and the time when the echo is received, the RT to the target. Especially with large bodies, such as planets, the difference between the shortest and longest delays can be large. The difference between those delays is the range dispersion, also known as radar depth, and the corresponding distance is a measure of the radius of the target. This also applies to asteroids, but being smaller bodies, the delay resolution plays an important role in being able to determine the size of those objects.

3) Doppler Shift Measurements: The Doppler shift is defined as the change in frequency observed in the received radar echo and caused by the interaction of the transmitted radar echo with the target. The relative motion between the target and the Earth-based GSSR produces a change in frequency and the frequency spectrum broadens. The amount of change depends on the relative velocity along the line of sight, i.e., Earth rotation, difference in orbital velocities between Earth and the target and the rotational motion of the target. Therefore, the spectral shape of the received echo provides information about the target. The center frequency of the received echo provides information on the Doppler frequency shift, which is a measure of the line-of-sight velocity or gross motion. The frequency spread is caused by target rotation. The received echo spectrum bandwidth is, therefore, a measurement of the target rotation velocity, where fast-rotating 
targets, i.e., large radial velocity, cause a wide frequency spread of the received echo and slow-rotation targets cause narrow frequency spreads. Orientation of the target, i.e., angle of the pole with respect to the line of sight from the target to the radar, has an impact on the Doppler shift measurement. When the target is orientated parallel with the spin axis, Doppler broadening is 0 . On the contrary, when the target is orientated perpendicular to the spin axis, Doppler broadening is maximum. The size of the target and the wavelength of the object also plays a role on the Doppler spectrum width.

Performing a Fourier transform on the range gates produces a frequency spectrum for each one of them and a delay-Doppler map is obtained. A delay-Doppler map is a way to resolve the composite return signal in terms of latitude and longitude of the target. Surface patches are uniquely identified except for the north-south hemispherical ambiguity, as the reflections from both of these surface points fall in the same delay-Doppler cell. By adding viewing geometry changes, or multistatic observations, this ambiguity can be resolved.

4) Polarization: The analysis of the polarization on planetary radar signatures brings insight into the nature of the surface, as it is a measure of its roughness. The GSSR can transmit a user-selectable polarizer. Upon reflection on the target surface those signals are reversed if the surface is smooth, so the OS polarization receives most of the power. If the surface is rough, SC polarization is also received. The rougher the surface is, the more signal is collected in the SC polarization, and less in the OS.

There have also been puzzling measurements where, given the presence of ice in the observed surfaces which makes them flat, while the echo was expected to appear in the OS polarization of the transmitted signal, it appeared on the SC polarization. That is the case of the Galilean satellites: Europa, Ganymede, and Callisto. Most theories and models point to the presence of structures that generate a double bounce, producing the SC polarization echo.

But generally speaking, from the power received at each polarization, the polarization ratio can be computed as the amount of SC polarization signal over the OS polarization signal. A polarization ratio near 0 is indication of smooth surface, while a polarization ratio near 1 or larger means the surface is rough on the order of the radar wavelength. Typically, most radar echoes have specular and diffuse components. The polarization ratio can be computed at each point in the frequency spectrum, making possible the location of smooth and rough areas within the target surface.

\section{EXISTING CAPABILITIES}

The GSSR has the capability to operate with different waveforms so it can be tailored to the specifics of the target and the investigation; one radar mode would not be suitable for the goals of all experimenters. Waveforms widely used on past experiments include $\mathrm{CW}, \mathrm{BPC}$, and chirp. Also, the transmitter/receiver configurations can be changed depending on the experiment, monostatic, or bistatic, as described in Section II-B2.

The CW mode transmits a constant radio frequency signal that has no time modulation. The amplitude and frequency

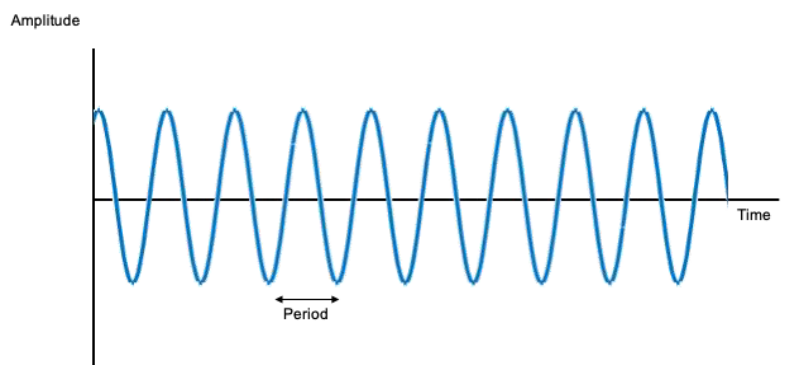

Fig. 4. CW waveform scheme.

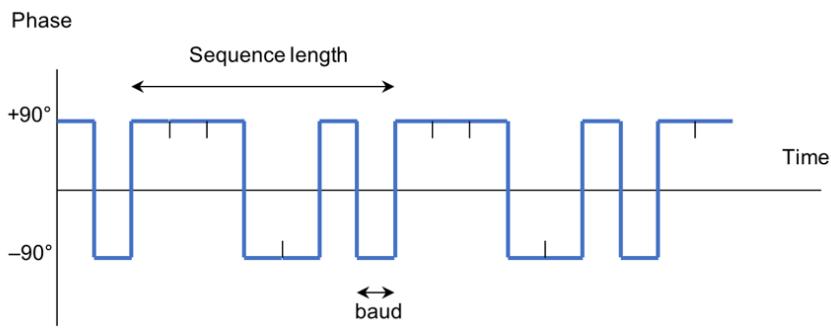

Fig. 5. BPC waveform modulation scheme showing the phase of the signal set to $+90^{\circ}$ and $-90^{\circ}$ according to a pseudo-random pattern.

bandwidth of the returned signal are used to determine the target characteristics such as radar cross section, rotation rate, and surface structure. The Doppler shift and frequency spread of the received $\mathrm{CW}$ echo varies with the radar scattering function of the target, and this is a function of the radius and rotation rate of the target. A CW waveform scheme is shown in Fig. 4.

The BPC mode is used for ranging measurements. A PN code is modulated on the carrier. Code lengths are long enough to cover the delay depth of the target, but not necessarily long enough to eliminate range ambiguity. Range ambiguities can be eliminated by transmitting pairs of codes with slightly different baud periods, usually 10 and $11 \mu \mathrm{s}$. To obtain the distance to the target, the radar system measures the RT travel time of the radar signal, from which the distance can be calculated. The range resolution depends on the time duration of one baud (chip rate), not the overall pulse. A subpulse is made short to give high-range resolution, while keeping the overall transmitted pulse longer, resulting in high average transmitted energy. A BPC waveform modulation scheme is shown in Fig. 5.

Currently, different configurations are used if objects' echoes permit them. Typically, the ranging starts with 10 and $11 \mu \mathrm{s}$ to get an absolute range measurement. If the echo is received successfully, $1,0.5,0.25$, and $0.125 \mu \mathrm{s}$ are used consecutively to further refine the range measurement of the object. For example, the resolution obtained from a $1-\mu$ s range gate size is about $150 \mathrm{~m}$. With $0.125 \mu \mathrm{s}$, the resolution is about $18.75 \mathrm{~m}$ and with $0.1 \mu \mathrm{s}$, the resolution is $15 \mathrm{~m}$. At higher baud resolutions, the sidelobe frequencies extend beyond what is safe for the klystrons. The best range resolution at Goldstone using BPC waveforms is obtained by using $0.05-\mu \mathrm{s}$ BPC as mentioned above, giving a resolution of $7.5 \mathrm{~m}$ on the existing DSS-14 high power transmitter and accomplished with the 


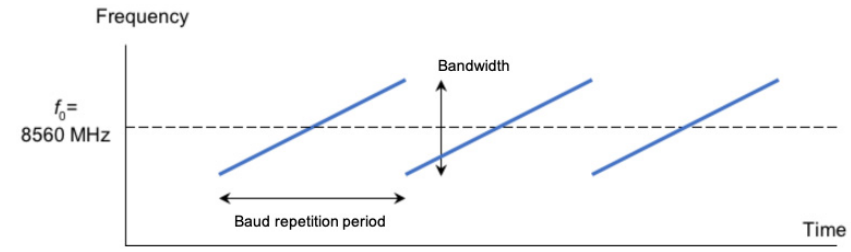

Fig. 6. Chirp waveform modulation scheme showing the frequency swept for every baud repetition period and the bandwidth used.

addition of a wide band low pass filter after the waveform generator.

Chirping or LFM is a more bandwidth efficient ranging signal used by GSSR in NEO observations, as it provides the best range resolution. In general, chirping correlation properties are slightly inferior to BPC, but the bandwidth efficiency allows for higher resolution ranging. A chirp waveform modulation scheme is shown in Fig. 6. The use of the chirp waveform for GSSR ranging is based on an informal report [32]. An internal JPL study [33] found that the practical bandwidth limit for the current Goldstone klystrons is $40 \mathrm{MHz}$, which leads to images at 3.75-m resolution [26]. The fundamental limitation to the range resolution is the bandwidth of the klystron power amplifiers through which the "chirp" waveform passes.

\section{GSSR ModifiCATIONS AND NEW CAPABILITIES}

During the past few years, in order to enhance the GSSR's ability to serve the planetary radar community, a number of modifications have been made.

1) A Higher Level of Automation: This modification enables the remote operation of the GSSR by the P.I. and the operator, reducing the level of complexity. By providing a remotely operable automation procedure, the number of scientific requests from P.I.s is expected to grow significantly, together with an increased in the number of P.I.s that the GSSR can attend. New capability: reduced complexity in remote operations.

2) A New Software-Driven Waveform Generator: The Arbitrary WFG (S-AWG). This modification enables versatility on the design of experiments allowing for a greater range of IF frequency selections and waveform definitions. With the new S-AWG, an increased number of scientific requests is expected since a variety of experiments are now possible. This will also contribute to the number of P.I.s that considers the GSSR for their investigations. New capability: increased experiment design versatility.

3) The Availability of the GSSR DCAR Receiver in Other Complexes, such as AO or GBT Observatories: This modification to the other observatories enhances their current capability and enables them to receive transmissions from DSN antennas, such as DSS-13 or DSS-14. The experiments benefit from the bigger size antennas, such as GBT's 100-m dish. Therefore, by equipping external observatories with a GSSR DCAR receiver, better range resolutions are achieved in bistatic experiments between the DSN and other observatories, i.e., $1.875 \mathrm{~m}$ when DSS-13 radiates an $80-\mathrm{MHz}$ chirp or $3.75 \mathrm{~m}$ when DSS-14 radiates a 40-MHz chirp, and in general, bistatic experiments are more scientifically successful. New capability: increased performance on bistatic radar experiments from GSSR to other observatories.

Section IV-A-IV-C detail the modifications made to the GSSR systems to obtain the abovementioned new capabilities.

\section{A. Automation of the GSSR Operation}

This section describes the effort carried out during the last years toward a higher level of automation in order to allow reduced complexity in remote operations. This section is organized into two parts. The first part presents an overview of the operator's responsibilities. The second part describes a general procedure using GSSR automated software package to assist the operator in achieving successful observations.

1) Definition of Operation: For a successful radar track, a GSSR operator is required to carry out a number of critical functions that ensure the success of the track and prevent the GSSR from being damaged. The following list provides the required actions:

1) Interface With DSN Operators: Automatic link builder (ALB) and link monitor and control (LMC). Communications between the GSSR operator and the assigned ALB operator (Goldstone/Madrid/Canberra) are maintained during precalibration and postcalibration. GSSR calibrations cannot begin until the antenna is fully configured (subreflector and DSN microwave elements) and any interlock issues are addressed to the operator. The LMC operator is then contacted to ensure that the initial predictions have been loaded correctly and to perform initial antenna movement from stow. Once the antenna is tracking, full control is given to the GSSR sequencer (Fig. 3) to change targets, offsets, and ephemeris files when needed, and communication with DSN operators is minimal.

2) Interface With Transmitter (XMT) Operator: Communication with the XMT operator is important as the transmitter calibration requires coordination with waveguide switch and drive activities. While the transmitter is being warmed up and calibrated, no other activities are conducted. The beam is solely controlled by the transmitter operator and cannot be turned on/off remotely. The waveguide switch is controlled by GSSR operator and must be properly configured for transmitter warm-up/ calibration.

a) During echo acquisition, the beam is usually turned on. The waveform output triggers the exciter ON or OFF for transmit-receive cycling. At this time, the whole control is under the GSSR operator, which supports the main rationale for the sequencer's automation.

b) The RT time caused by the electronic delay is calculated for each waveform before Doppler precompensation is applied. This process is called local loopback calibration. It is achieved by leaking a small amount of energy that goes from the waveform generator all the way up to the subreflector via the transmit path and bounces back 
to the back-end receiver. The time difference of the signal leaving the waveform generator to the signal collected by the receiver is the system's overall electronic delay. The loopback delay calculated with higher resolution waveform will always generate more accurate delay. The best number that can be obtained is referred as to absolute ranging.

c) The local loopback information also serves as a configuration check to confirm the transmit signal is what the receiver expects.

3) Control and Monitor Antenna Movement: Antenna pointing to the target is critical for the success of the radar observation. During transmission, the antenna concentrates its energy into a shaped beam that points in the direction of the desired target. At DSS-14, the pointing is controlled by a so-called "source" file that consists of three sets of timestamped right ascension (RA) and declination (DEC) pairs of the target. With proper interpolation, the target can be tracked precisely. In addition, DSS-14's antenna control system also provides the possibility to set an offset which enables the capability to apply minor changes to its pointing. The GSSR uses this offset to adjust for the pointing difference produced when switching from the transmit position to the receive position, and vice versa. It is important to set pertinent antenna movement and offsets by setting antenna pointing and automotion, such as boresights and uploaded pointing, which can also be updated in real time. In order to prevent interlock, waveguide switches need to be controlled and sequenced. Note that to ultimately prevent damage to high-power transmitter, quick decisions are often required.

4) Monitor, Calibrate, and Record System Temperature (Tsys): It is required to calibrate Tsys during precalibration and monitor it throughout the observation. Tsys can change due to weather conditions, elevations, or beam obstructions. The information is always logged and can be used for radar cross-section (RCS) calculation, incoherent summing, and model shaping.

5) Control Signal Routing Path: Properly patch the uplink and downlink signal cables for accurate transmit/receive configuration.

6) Configure and monitor P.O. and waveform generator with ephemeris for Doppler precompensation. It is required to properly configure the synthesizer, which must be referenced, to monitor instantaneous Doppler frequency versus expected frequency and understand the ephemeris file.

7) Control back-end receiver with filter, level, and bandwidth settings. It is required to select appropriate filter and attenuation based on the transmitted waveform, properly set up coupling (ac/dc), and properly configure receiver's LOs $(2 \times$ IF center) and levels.

8) Command data acquisition and recording, including synthesizers for ADC.

9) Calibrate absolute ranging with loopback.

10) Real-time image processing and presentation.
11) Revise Ephemeris File: This step is carried out if the P.I. requests it and allows for fast turn-around on reconfiguration of the predict file, if provided.

12) Dynamic Decision-Making: A dynamic process that allows real-time decisions helps scientists to make optimal decisions in maximizing the scientific return of the observation.

From the list of critical actions and the coordination needed between DSN operators, GSSR operator, and P.I.s, it is apparent that the operation of the GSSR is very complex. Next, we present the operation procedure and the efforts made to ease the role of the GSSR operator during a track.

2) Operation Procedure: During the last years, a GSSR automated software package has been designed to increase the efficiency in the procedures during a radar track, and improve the communication between GSSR operator, antenna operator, transmitter operator, and the P.I.s. Fig. 7 shows the overall operation procedure. Before each experiment, the P.I.s need to submit a track request to DSN scheduler. Once the track is approved, the P.I.s need to furnish target information (such as ephemeris and antenna transmitter/receiver offsets) to the operator. During the track day, the P.I.s can observe the track by logging into the GSSR back-end machine, where they will receive real-time processed images. From the images, P.I.s can make decisions for another suitable configuration based on the available SNR. After several iterations, a desired configuration can be derived for the best scientific value of the target. Each configuration will be input into the GSSR automated software package, which can be controlled by a GSSR operator or the P.I.s themselves. For simplicity, for the following description, we refer to this person as an operator. Various P.I.s and GSSR operators (when present) usually communicate and discuss through available communication tools, such as Skype or WebEx. The discussions that occur there are often vital to the success of the track. These discussions are often logged or archived as well. The operator needs to coordinate with the DSN operator to build an LMC. The operator also needs to work with the XMT operator for transmitter calibration. Extended details on what is conducted in the GSSR automated software package are shown in Fig. 8.

From the diagram shown in Fig. 8, the GSSR automated software package starts by initializing the GSSR and starting the master sequencer GUI [shown in Fig. 15(a) in Appendix A]. A number of actions are carried out during initialization, such as creating directories for the particular track, setting the target name, establishing the RT time, locating the predicts file, and filling antenna pointing information. After everything is set up, the operator communicates to the LMC that the link is ready to be assigned. After approval, all antenna function can be remotely controlled by the master sequencer through EAC. The operator then proceeds to measure the system parameters (Yfactor, system temperature, and transmitted power), which are recorded into a $\log$ file for archiving. If needed, the operator can proceed with the boresight calibration. The system environment is also read and stored into a file. The next step is to setup the antenna control and ask for LMC approval for controlling the antenna and for 


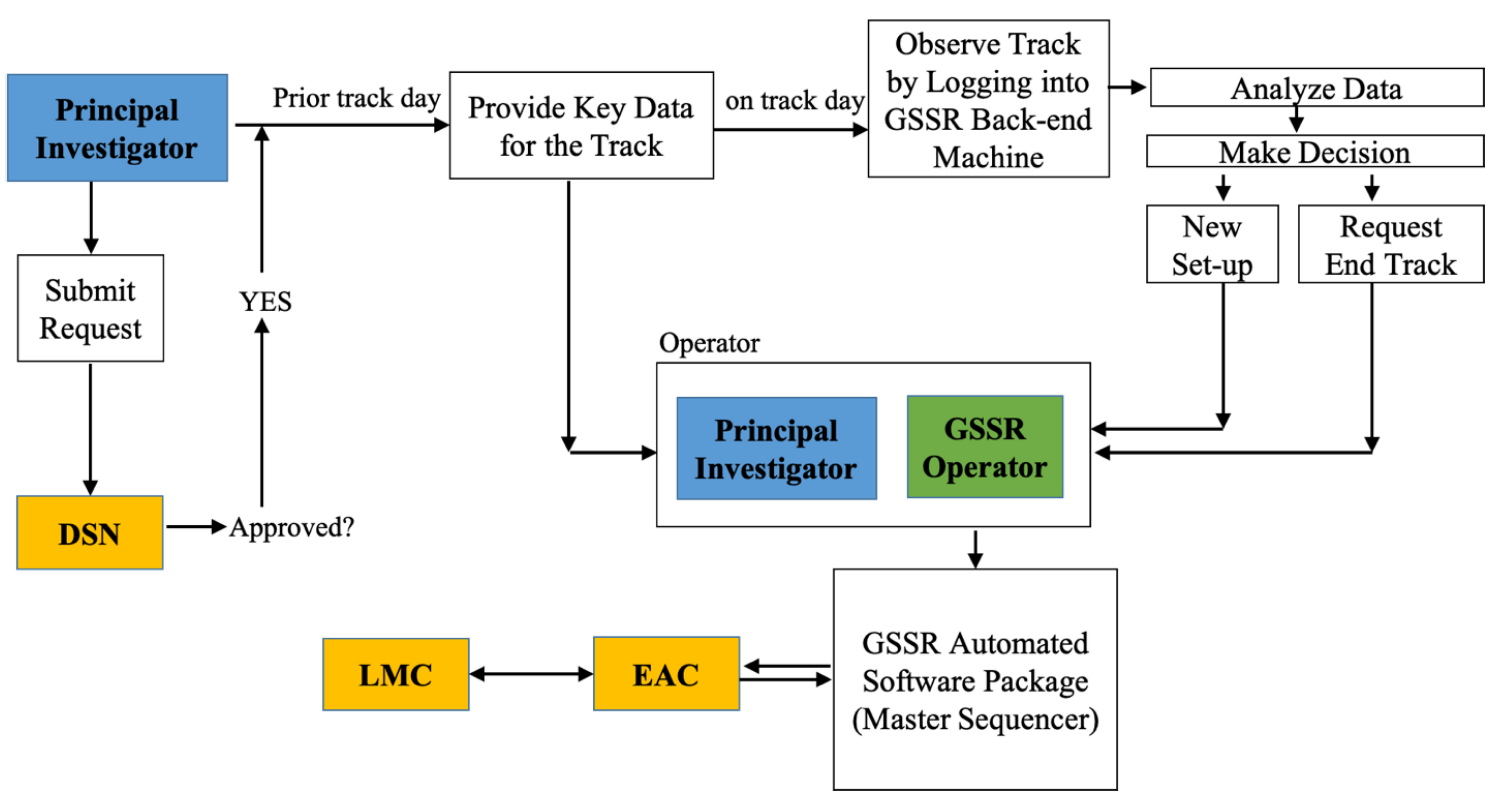

Fig. 7. Radar track operation procedure diagram.

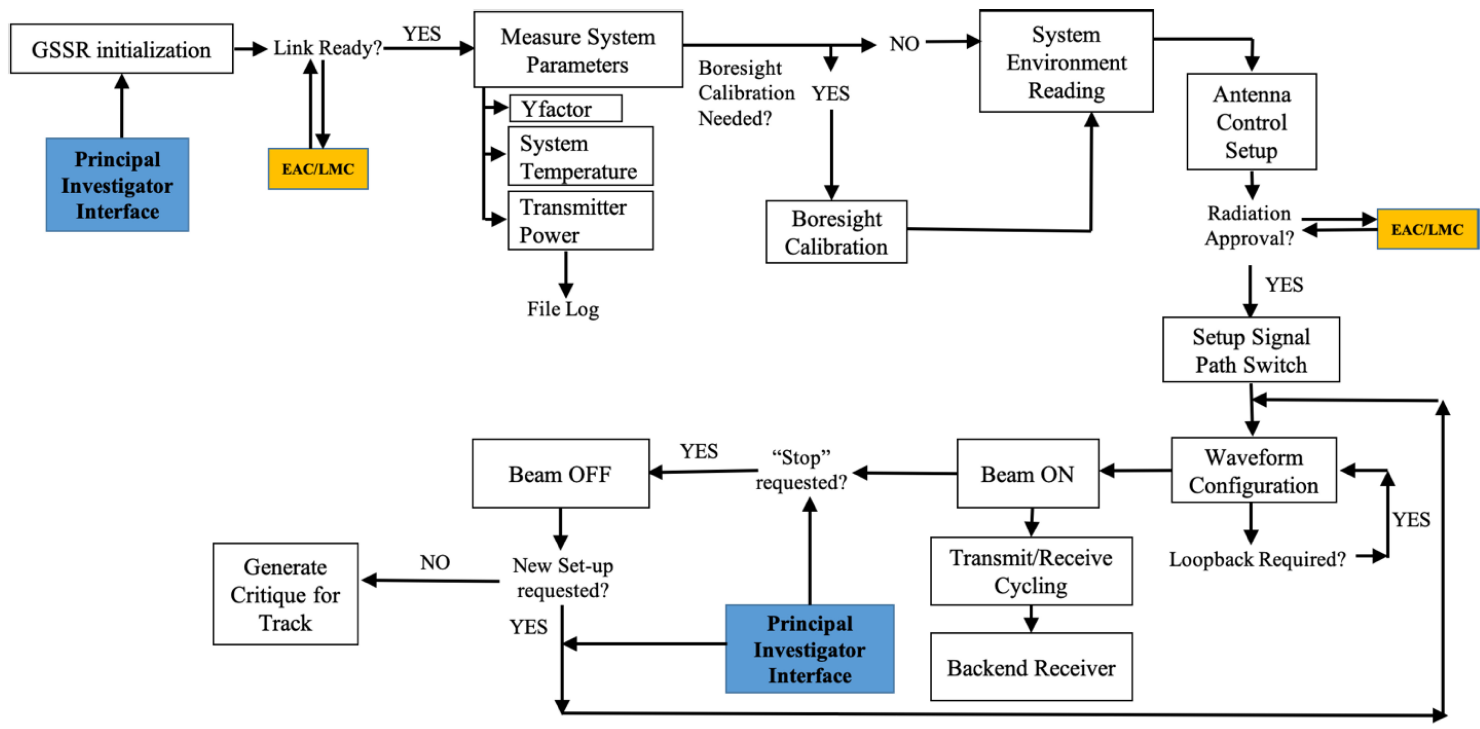

Fig. 8. GSSR automated software package diagram.

radiation, which once approved will assign control through the EAC.

The operator will properly set up the signal path switch, such as channels, polarizations, bandpass filters, and attenuation, and then proceeds with the system configuration, i.e., the receiver configuration, the waveform configuration, and loopback settings, if/when needed. Given that the operator already obtained radiation approval, the beam can be turned on.

At any time, as shown in both Figs. 7 and 8, the P.I.s can stop the transmission, either because the track is ending or because there is a need to change the system configuration. Note that P.I.s are specifically mentioned here, as this task is specific to them. When the operator receives the request from the P.I.s, the operator stops the transmitter. If there is a request for a new setup, the operator repeats the steps from system configuration to transmission. (There is no need to get LMC approval after the initial approval.) If there is a request to end the track, the operator turns the beam off, with an option for stopping it gracefully (waiting until the end of the receive cycle), or without further delay. In both cases, the operator communicates with the transmitter operator to turn the transmitter off. The operator then sets the GSSR and the antenna into a safe position and disarms the systems. Once the track is done, the operator prepares for a DSN critique report, and gets it signed and sent to all concerned parties.

\section{B. Arbitrary Waveform Generator $(S-A W G)$}

This section describes the recent effort carried out toward the development of the new software-driven Signatec Arbitrary WFG (S-AWG) in order to allow more versatility in experiment. The new S-AWG, as being software based, can run 


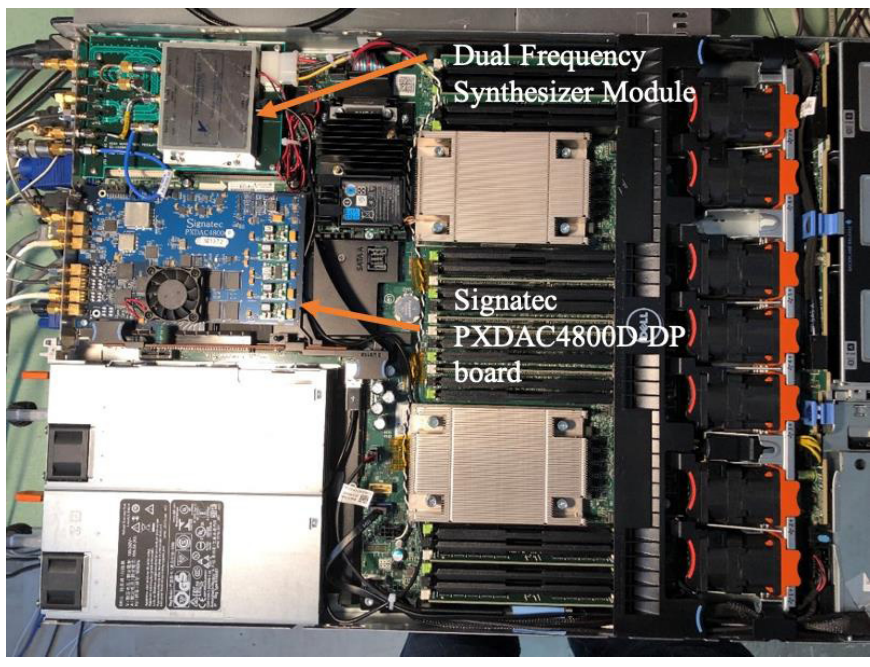

Fig. 9. S-AWG is a $1 \mathrm{U}$ rack mount Dell computer with two 44-core CPUs.

in a wide spectrum of machines from a Linux machine with 8 cores (16 threads) to a dual Intel Xenon server with 44 cores (88 threads). The P.O./waveform generator used for years at the GSSR is capable of producing CW, BPC, and chirp waveforms. This waveform generator is an FPGA-based hardware that has been in commission since 2010. Its design has been unmaintainable due to the lack of vendor and designer support and the availability lack of hardware parts. In addition, the design is based an on-board crystal oscillator and only three frequencies can be synthesized: 325,460 , and $560 \mathrm{MHz}$. In order to add versatility to the waveform definition and the frequency selection, a new waveform generator has been designed: the S-AWG based on a Signatec PXDAC4800D-DP board. The S-AWG is a software-driven system that allows the custom selection of the IF frequency from $50 \mathrm{MHz}$ to $1 \mathrm{GHz}$. In addition, any arbitrary waveform can be configured: from the currently used CW, BPC, and Chirp to other types of waveforms, such as single sideband band (SSB) and quadrature amplitude modulation (QAM), among the many possibilities. The S-AWG computer is shown in Fig. 9.

In Fig. 9, the Signatec PXDAC4800D-DP board and the Valon Technology 5009 Dual Frequency Synthesizer Module are marked. Appendix B includes specifications for these two key components.

In addition, the GSSR automated software package also includes a control panel for the S-AWG (Fig. 10).

Fig. 10 shows how a user can configure the S-AWG for the currently defined waveforms. As it can be seen, the configuration selected is for $\mathrm{CW}$ with an offset of $500 \mathrm{~Hz}$, but examples for BPC and chirp configurations are also given. The development to have user-defined or flexible waveform is still ongoing when this document is being written.

\section{Equip GBT and AO With GSSR Back-end DCAR Receivers}

This section describes the effort carried out during the last years toward bringing the GSSR back-end DCAR receiver to other complexes in order to facilitate an increased performance

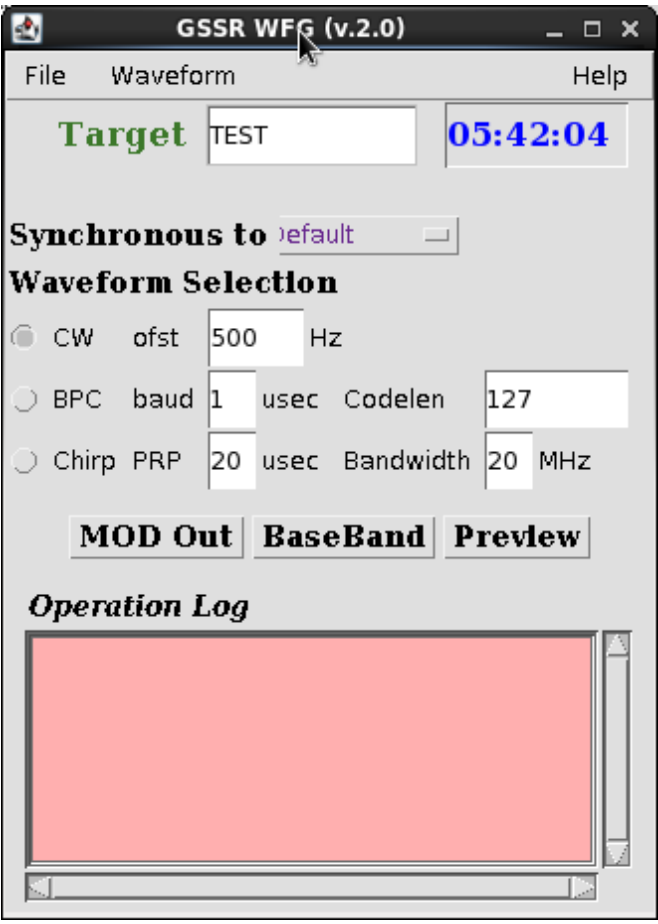

Fig. 10. GSSR S-AWG control GUI panel.

on bistatic radar experiments from GSSR to other observatories. The Robert C. Byrd GBT in Green Bank, West Virginia, is the world's largest fully steerable radio telescope. The AO in Arecibo, Puerto Rico, was-until recent eventsthe world's second largest single-aperture telescope. Even though AO's size was next to the Five-hundred-meter Aperture Spherical radio Telescope (FAST) in China, the AO power capability was superior with 1-MW high-power transmitter, while powerful transmitters are too large and heavy for FAST's small receiver cabin. The GBT observatory can carry out bistatic radar measurements when combined with transmitting DSS-14 or DSS-13 antennas. DSS-13 antenna is located just 15 miles from DSS-14 and has a 34-m dish.

GSSR back-end and DCAR receivers were installed on $\mathrm{AO}$ and GBT telescope sites. In addition to the hardware, a software package was delivered for easing configuration of the DCAR receivers. Cooperation among these sites helped carry out some very interesting observations. As an example, in June 2018, NEO 2017YE5 was successfully observed in becoming very relevant to the press and the public in general: "new observations by three of the world's largest radio telescopes have revealed that an asteroid discovered last year is actually two objects, each about 3000 feet $(900 \mathrm{~m})$ in size, orbiting each other" (source: https://www.naic.edu/ao/node/1024\#). More information on this particular finding and other can be found in the echo.jpl.nasa.gov website, where all GSSR and other observatories observations are recorded and described. Figs. 11 and 12 show images of the front panel and back panel of the GSSR back-end DCAR receivers installed in the external observatories.

Some of the specifications of the GSSR back-end DCAR are as follows: 
TABLE V

Suggested DCAR CONFIguration BASEd ON THE TRANSMitTEd WAVEForm

\begin{tabular}{|c|c|c|c|c|c|}
\hline Configuration & $\begin{array}{c}\text { Synthesizer } \\
\text { (Valon \& Signatec) }\end{array}$ & $\begin{array}{c}\text { Division } \\
\text { (Signatec) }\end{array}$ & $\begin{array}{l}\text { Effective } \\
\mathrm{F}(\mathrm{s})\end{array}$ & $\begin{array}{l}\text { LPF Selection } \\
\text { (Agile Receiver) }\end{array}$ & $\begin{array}{l}\text { Range Resolution } \\
(\mathrm{m})\end{array}$ \\
\hline $\mathrm{CW}$ (up to $1 \mathrm{MHz}$ ) & 200 & 32 & 6.25 & $1.3 \mathrm{MHz}$ & N/A \\
\hline ВРC $10-11 \mu \mathrm{s}$ & 256 & 32 & 8 & $1.3 \mathrm{MHz}$ & 3000 \\
\hline $\mathrm{BPC} 1 \mu \mathrm{s}$ & 256 & 32 & 8 & $1.3 \mathrm{MHz}$ & 300 \\
\hline ВРC $0.5 \mu s$ & 256 & 32 & 8 & $2.7 \mathrm{MHz}$ & 150 \\
\hline BPC $0.25 \mu \mathrm{s}$ & 256 & 16 & 16 & $5 \mathrm{MHz}$ & 75 \\
\hline BPC $0.125 \mu \mathrm{s}$ & 256 & 8 & 32 & $9 \mathrm{MHz}$ & 37.5 \\
\hline ВРС $0.1 \mu s$ & 320 & 8 & 40 & $11 \mathrm{MHz}$ & 30 \\
\hline $\mathrm{BPC} 0.0625 \mu \mathrm{s}$ & 256 & 4 & 64 & $17 \mathrm{MHz}$ & 18.75 \\
\hline BPC $0.05 \mu \mathrm{s}$ & 320 & 4 & 80 & $21 \mathrm{MHz}$ & 15 \\
\hline BPC $0.03125 \mu s$ & 256 & 2 & 128 & $33 \mathrm{MHz}$ & 9.375 \\
\hline LFM $10 \mathrm{MHz}$ & 320 & 16 & 20 & $6 \mathrm{MHz}$ & 15 \\
\hline LFM $20 \mathrm{MHz}$ & 320 & 8 & 40 & $11 \mathrm{MHz}$ & 7.5 \\
\hline LFM $30 \mathrm{MHz}$ & 320 & 4 & 80 & $21 \mathrm{MHz}$ & 3.75 \\
\hline LFM $40 \mathrm{MHz}$ & 320 & 2 & 160 & $41 \mathrm{MHz}$ & 1.875 \\
\hline
\end{tabular}

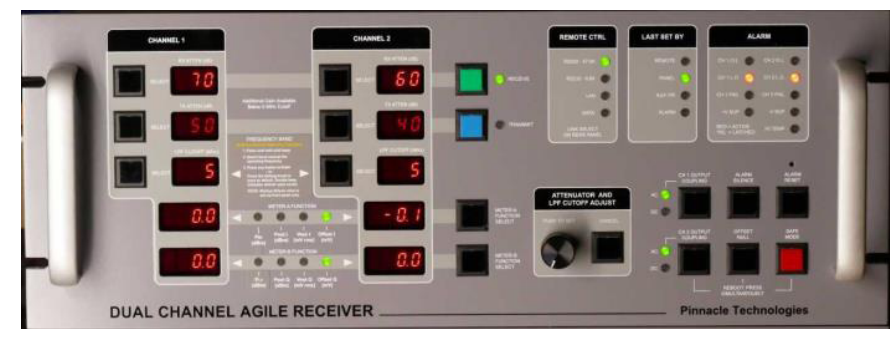

Fig. 11. Photograph of the front panel of DCAR. An operator can configure DCAR from the panel or via the GUI software provided.

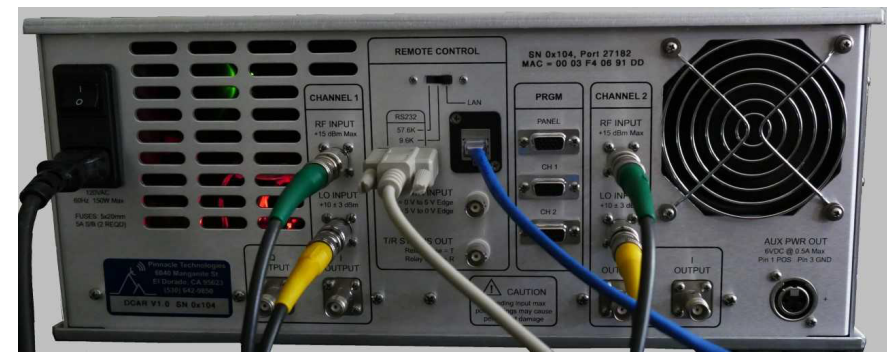

Fig. 12. Photograph of the back panel of DCAR. The RS232 interface allows the sequencer to protect the receiver while cycling between transmit and receive for a monostatic track.

1) IF (50-1000 MHz) downconverter;

2) Individually configurable for single or dual polarization inputs;

3) Programmable attenuation ranges from 0 to $70 \mathrm{~dB}$;

4) Programmable low-pass filter (LPF) bandwidth ranges from $160 \mathrm{kHz}$ to $64 \mathrm{MHz}$;

5) Selectable ac- or dc-coupled;

6) Built-in power meters for diagnostics;

7) TTL-level XMT/RCV switching for monostatic observation;

8) Support both RS232 and LAN interfaces with a fully configurable front panel.

Table V presents a summary of the GSSR back-end DCAR configurations that can be implemented depending on the transmitted waveform.

There are a great variety of options for the different waveform types that could be used to achieve different

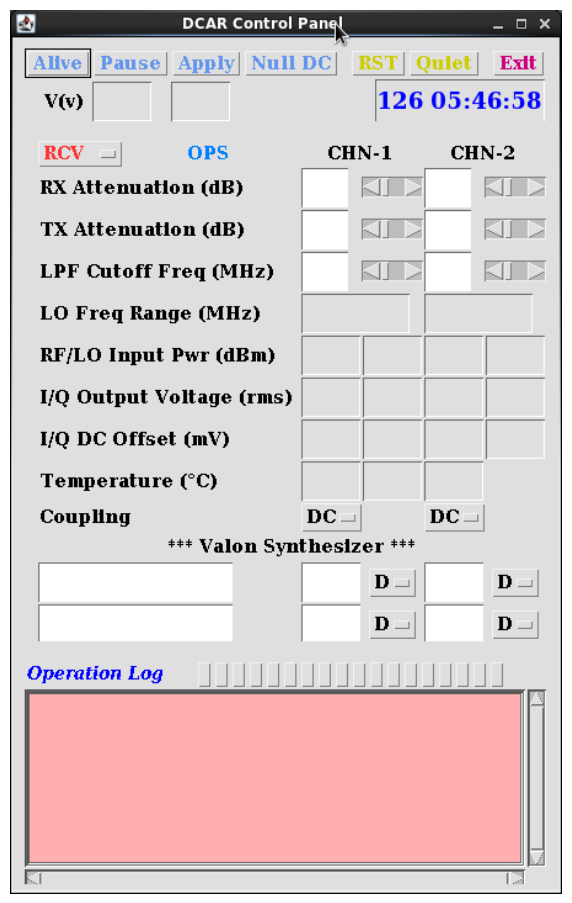

Fig. 13. DCAR configuration of the GUI panel from the GSSR automated software package.

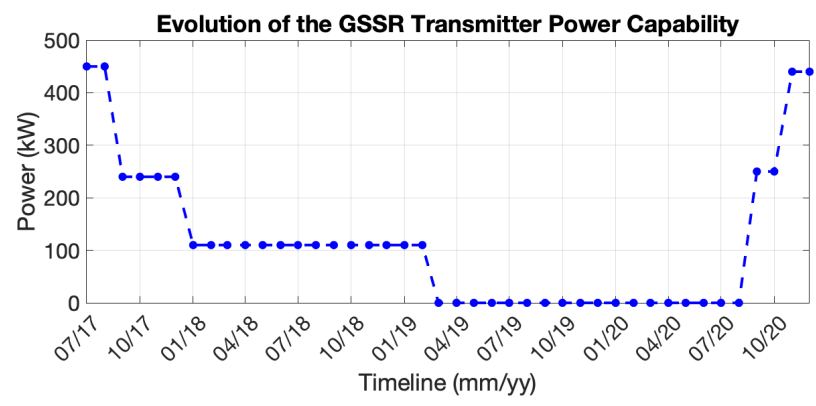

Fig. 14. GSSR transmitter power capability timeline since 2017 to current capability after S/N 111 and S/N 112 klystrons installation.

range resolutions. The GSSR automated software package also includes a configuration GUI panel that allows those configurations (Fig. 13). 


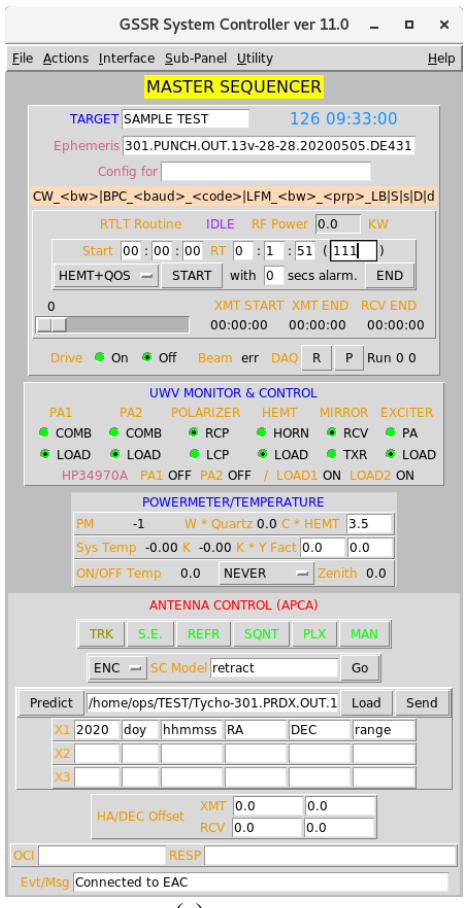

(a)

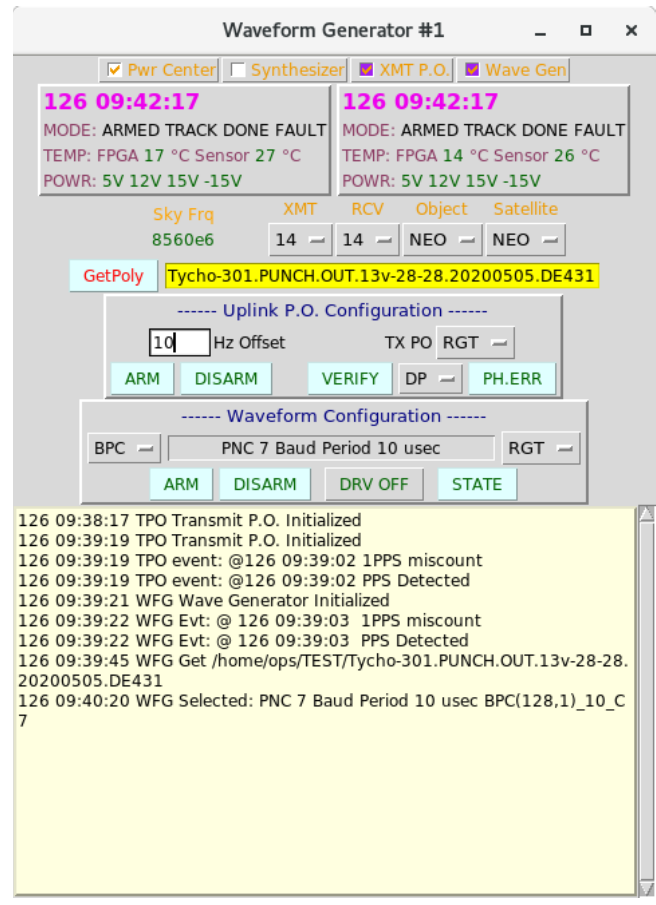

(b)

Fig. 15. GSSR automated software package panels. (a) Master sequencer GUI and (b) waveform generator GUI panel.

As can be seen from Fig. 13, the GUI allows the operator to fully control and monitor DCAR operation status. An operator can set the correct LPF, set the level based on the power meter reading, and change the LO frequency to match the IF frequency. Signal coupling is also controllable. As mentioned above, along with the hardware, this software and the signal processing software were also provided to the GBT and the AO.

\section{RETURN TO THE SERVICE OF GSSR}

JPL and CPI collaborated over the past five years to redesign GSSR's klystrons for increased reliability and improved performance. The prototype of the redesigned klystron $\mathrm{S} / \mathrm{N}$ 110 was accordingly developed and tested for a year.

The GSSR had full power ( $\sim 450 \mathrm{~kW})$ until September 2017, when one of the previous klystrons failed. Since that moment, the GSSR started operating at 50\% power, using only S/N 110. Another failure made the GSSR operate at only $110 \mathrm{~kW}$ using a spare klystron for over a year, in order to prevent the failure of the last functioning klystron. In March 2019, the last working klystron failure made the GSSR nonoperative for nearly one year and three months.

CPI delivered a new klystron (S/N 111) in June 2020, which was then installed in the DSS-14 antenna and successfully performed its first radar tracks in September 2020 with a power capability of $250 \mathrm{~kW}$. A second klystron (S/N 112) was completed by CPI and installed on the GSSR in November 2020 which has allowed the return of the GSSR to its full $\sim 440 \mathrm{~kW}$ operation. Fig. 14 shows a power capability timeline of the klystrons and power capabilities of the GSSR from
2017 to the present capability after the successful installation of klystron S/N 111 and klystron S/N 112.

\section{CONCLUSION}

The GSSR is a very powerful asset that for several decades has served in the radar scientific community by supporting experiments that looked at a great variety of solar system bodies. From its creation, the GSSR has supported observations of Venus, Mercury, Moon, Mars, the Galilean satellites, and Titan. More recently, the use of the GSSR has been focused primarily in the tracking and characterization of asteroids. In this article, we have presented a number of modifications that have enabled new capabilities: reduced complexity in remote operations, increased experiment design versatility, and increased performance on bistatic radar experiments from GSSR to other observatory complexes. These new capabilities have brought the GSSR closer to the needs of the scientific community showing increased performances of the science return. Thanks to the current GSSR capabilities, and the return to service at full-power capabilities in November 2020, the expected outcome is an increase in scientific experiments as well as an increase on the number of P.I.s employing the GSSR in their scientific investigations. An increased number of investigations will increase the scientific return associated to the GSSR and will help toward increasing our knowledge on planetary science. Given the vision toward setting astronauts in the Moon, the recent successful commercial launch to the International Space Station, and the forecast increase of space traffic around Earth, the Moon and potentially Mars, in the upcoming years, it becomes of importance to improve the capabilities for detecting and monitoring small solar system 


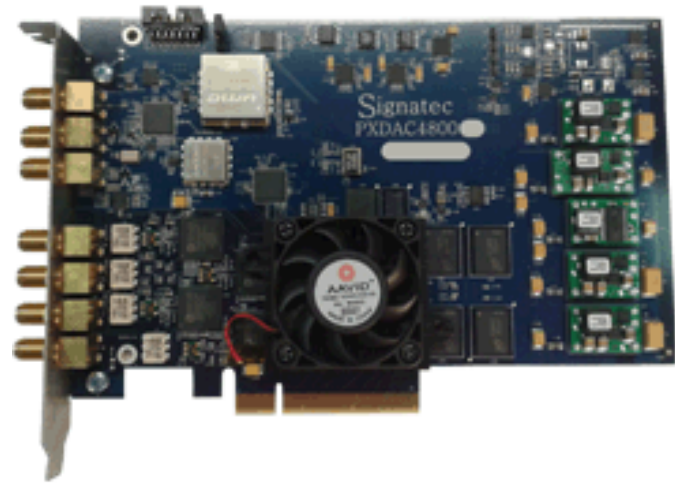

Fig. 16. Signatec PXDAC4800D-DP board.

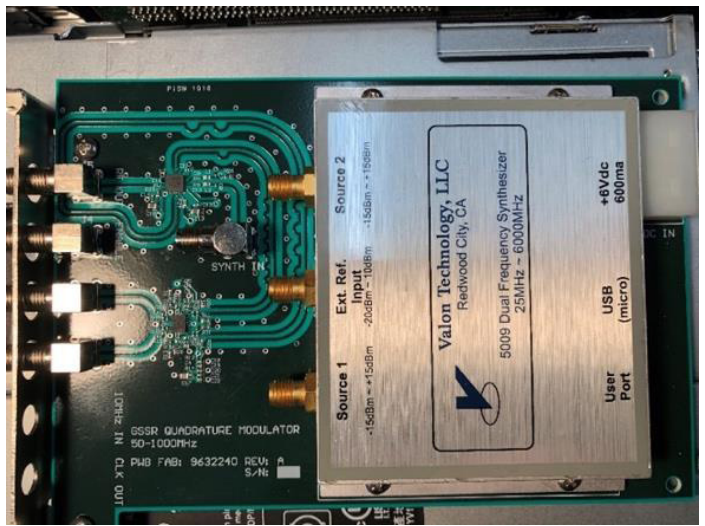

Fig. 17. Valon Technology 5009 Dual Frequency Synthesizer Module.

bodies. The GSSR is a key asset that accomplishes both science objectives, helping in the understanding of the solar system, and planetary defense objectives, helping toward the critical task of improving asteroid or debris impact predictions.

\section{APPENDIX A}

This appendix provides an illustrative example of the GSSR automated software package GUI (Fig. 15).

Fig. 15(a) and (b) shows the GUI panel for the master sequencer and the GUI panel for the waveform generator, respectively. In particular, Fig. 15(b) shows an example of the configuration set for the monostatic tracking of an NEO with a 10- $\mu$ s BPC waveform.

\section{APPENDIX B}

This appendix includes the specifications for the two key components of the S-AWG: the Signatec PXDAC4800D-DP board and the Valon Technology 5009 Dual Frequency Synthesizer Module.

The specifications of the Signatec PXDAC4800D-DP board (see Fig. 16) are as follows:

1) Up to $900 \mathrm{MB} / \mathrm{s}$ continuous sustained transfer via PCI Express x8 Bus;

2) Four dc-coupled 14-bit or 8-bit DAC outputs;

3) 14-bit $1.2 \mathrm{GS} / \mathrm{s}$ for two channels or $600 \mathrm{MS} / \mathrm{s}$ for four channels;

4) 8-bit $1.2 \mathrm{GS} / \mathrm{s}$ for four channels;

5) 1-GB DDR2 RAM;

6) Based on analog devices AD9736 DAC;
7) Output ranges from 400 to $1470 \mathrm{mV}$;

8) Bandwidth up to $400 \mathrm{MHz}$ (ac-coupled) or $590 \mathrm{MHz}$ (dc-coupled).

The specifications of the Valon Technology 5009 Dual Frequency Synthesizer Module and the quadrature modulator (see Fig. 17) are as follows:

1) Frequency range: $23-6000 \mathrm{MHz}$;

2) Output power: $+15 \mathrm{dBm}$ and can be adjusted in $0.5-\mathrm{dB}$ steps with 31.5-dB step attenuator;

3) Two independent sources phase locked to the same internal or external reference;

4) USB and TLL serial interface;

5) Flash-based, nonvolatile memory.

\section{ACKNOWLEDGMENT}

This work was carried out at the Jet Propulsion Laboratory, California Institute of Technology, under a contract with the National Aeronautics and Space Administration. Government sponsorship acknowledged.

\section{REFERENCES}

[1] D. C. Elder, "Something of value: Echo and the beginnings of Satellite communications," in Beyond the Ionosphere: Fifty Years of Satellite Communication, A. J. Butrica, Ed. Washington, DC, USA: NASA, 1997, ch. 4.

[2] A. F. C. Haldemann, D. O. Muhleman, B. J. Butler, and M. A. Slade, "The western hemisphere of Venus: $3.5 \mathrm{~cm}$ dual circular-polarization radar images," Icarus, vol. 128, no. 2, pp. 398-414, Aug. 1997.

[3] R. F. Jurgens et al., "Radar observations of tinatin planitia: Goldstone 1988 observations of Venus," in Lunar and Planetary Science XXI, no. 1303. Houston, TX, USA, 1990, pp. 593-594.

[4] M. A. Slade, S. Zohar, and R. F. Jurgens, "Venus: Improved spin vector from Goldstone radar observations," Astronomical J., vol. 100, no. 4 pp. $1369-1374,1990$

[5] D. O. Muhleman, B. J. Butler, and M. A. Slade, "Radar imaging of the ice deposits on Mercury's poles," in Proc. 25th Lunar Planetary Sci. Conf., Houston, TX, USA, 1994, pp. 951-952.

[6] B. J. Butler, D. O. Muhleman, and M. A. Slade, "VLA/Goldstone 3.5-cm radar observations of Mercury in 1994: South polar and other results," Bull. Amer. Astonomical Soc., vol. 26, p. 1106, Jun. 1994.

[7] J. K. Harmon and M. A. Slade, "Radar mapping of mercury: Full-disk images and polar anomalies," Science, vol. 258, no. 5082, pp. 640-643, Oct. 1992.

[8] J. L. Margot, D. B. Campbell, R. F. Jurgens, M. A. Slade, and N. J. Stacey, "High resolution topographic maps of the lunar south pole," Bull. Amer. Astronomical Soc., vol. 29, p. 986, Jul. 1997.

[9] J. L. Margot, D. B. Campbell, R. F. Jurgens, M. A. Slade, and N. J. Stacey, "The topography of the lunar polar regions from Earthbased radar interferometry," in Proc. 29th Lunar Planetary Sci., 1998, pp. $1845-1846$.

[10] M. A. Slade, B. J. Butler, J. K. Harmon, R. F. Jurgens, and A. F. C. Haldemann, "Radar full-disk imaging and topography of Mars during the 1999 opposition," in Proc. 29th Lunar Planet. Sci. Houston, TX, USA: Lunar and Planetary Institute, 1998, p. 1340.

[11] J. K. Harmon, M. A. Slade, and R. S. Hudson, "Mars radar scattering: Arecibo/Goldstone results at $12.6-$ and $3.5-\mathrm{cm}$ wavelengths," Icarus, vol. 98, no. 2, pp. 240-253, 1992.

[12] A. F. C. Haldemann, D. L. Mitchell, R. A. Jurgens, M. A. Slade, and D. O. Muhleman, "Mars Pathfinder landing site assessment with Goldstone delay-Doppler and CW radar experiments," J. Geophys. Res., vol. 102, pp. 4097-4106, 1997.

[13] S. J. Ostro et al., "Europa, Ganymede, and Callisto: New radar results from Arecibo and Goldstone," J. Geophys. Res. Planets, vol. 97, no. E11, 1992, pp. $18227-18244$.

[14] D. O. Muhleman, A. W. Grossman, M. A. Slade, and B. J. Butler, "Titan's radar reflectivity and rotation," Bull. Amer. Astronomical Soc., vol. 25, p. 1099, Jun. 1993.

[15] D. O. Muhleman, A. W. Grossman, M. A. Slade, and B. J. Butler, "The surface of Titan and Titan's rotation: What is radar telling US?" Bull. Amer. Astronomical Soc., vol. 24, pp. 954-955, Jun. 1992. 
[16] L. A. M. Benner et al., "Radar observations of asteroid 2063 Bacchus," Icarus, vol. 139, no. 2, pp. 309-327, Jun. 1999.

[17] D. L. Mitchell, R. S. Hudson, S. J. Ostro, and K. D. Rosema, "Shape of asteroid 433 Eros from inversion of Goldstone radar Doppler spectra," Icarus, vol. 131, no. 1, pp. 4-14, 1998.

[18] D. L. Mitchell et al., "Radar observations of asteroids 1 Ceres, 2 Pallas, and 4 Vesta," Icarus, vol. 124, no. 1, pp. 113-133, Nov. 1996.

[19] C. Magri et al., "Radar observations and a physical model of Asteroid 1580 Betulia," Icarus, vol. 186, no. 1, pp. 152-177, Jan. 2007.

[20] L. A. M. Benner et al., "Radar observations of Asteroid 1999 JM8," Meteoritics Planet. Sci., vol. 37, no. 6, pp. 779-792, Jun. 2002

[21] L. A. M. Benner et al., "Radar images of asteroid $100085(1992$ UY4)," presented at the IAU Symp., Prague, Czech Republic, Aug. 2006. [Online]. Available: http://hdl.handle.net/2014/39808

[22] S. J. Ostro et al., "Radar imaging of binary near-Earth asteroid (66391) 1999 KW4," Science, vol. 314, no. 5803, pp. 1276-1280, Nov. 2006.

[23] M. Brozović, "Goldstone and Arecibo radar observations of (99942) Apophis in 2012-2013," Icarus, vol. 300, pp. 115-128, Jan. 2018.

[24] K. J. Lawrence et al., "Arecibo and goldstone radar images of near-Earth asteroid (469896) 2005 WC1," Icarus, vol. 300, pp. 12-20, Jan. 2018.

[25] A. Virkki, E. Zubko, M. C. Nolan, E. S. Howell, L. A. M. Benner, and J. K. Harmon, "Decimeter-scale particle characterization in the coma of 73P/Schwassmann-Wachmann 3 using dual-wavelength radar observations," Icarus, vol. 325, pp. 94-104, Jun. 2019.

[26] M. A. Slade, L. A. M. Benner, and A. Silva, "Goldstone solar system radar observatory: Earth-based planetary mission support and unique science results," Proc. IEEE, vol. 99, no. 5, pp. 757-769, May 2011.

[27] J. D. Dvorsky, N. A. Renzetti, and D. E. Fulton, "The Goldstone solar system radar: A science instrument for planetary research," NASA, Washington, DC, USA, Tech. Rep. JPL Publication 92-29, 1992.

[28] S. J. Ostro, Asteroid Radar Astronomy. Tucson, AZ, USA: Asteroids III, Univ. Arizona Press, 2002, p. 152.

[29] L. Teitelbaum, R. Liou, Y. Vodonos, J. Velazco, K. Andrews, and D. Kelley, "The klystron engineering model development (KEMD) task-A new design for the Goldstone solar system radar (GSSR)," Jet Propuls. Lab. Interplanetary Netw., Prog. Rep. 42-210, Aug. 2017.

[30] S. D. Slobin. (Accessed: Apr. 29, 2020). 101 70-m Subnet Telecommunications Interfaces. DSN nos. 005-810, 101, Rev. G Issue Date: September 4, 2019 JPL D-19379; CL\#19-5458. [Online]. Available: https://deepspace.jpl.nasa.gov/dsndocs/810-005/101/101G.pdf

[31] P. Potter, "The application of the cassegrainian principle to ground antennas for space communications," IRE Trans. Space Electron. Telemetry, vol. SET-8, no. 2, pp. 154-158, 1962.

[32] L. Margot, "Planetary radar astronomy with linear FM (Chirp) waveforms," Nat. Astron. Ionosphere Center, Internal Arecibo Memo, Tech. Rep., Jun. 2000.

[33] K. J. Quirk and M. Srinivasan, "GSSR waveforms for lunar observations," Jet Propuls. Lab., Interplanetary Netw., Prog. Rep. 42-192, Feb. 2013.

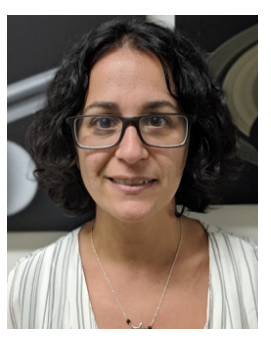

Nereida Rodriguez-Alvarez (Senior Member, IEEE) received the B.S. and M.S. degrees in telecommunications and the Ph.D. degree in remote sensing from the Universitat Politecnica de Catalunya, Barcelona, Spain, in 2005, 2007, and 2011, respectively.

She was a Postdoctoral Researcher with the National Oceanographic and Atmospheric Administration (NOAA), Boulder, CO, USA, the Atmospheric Science Department, Colorado State University, Fort Collins, CO, USA, and Purdue University, West Lafayette, IN, USA. She was part of the Cyclone Global Navigation Satellite System (CYGNSS) Science Team, for three years. She specializes on bistatic radar techniques for sensing the Earth employing signals of opportunity, through Global Navigation Satellite System-Reflectometry (GNSS-R), and has contributed to the retrieval of land geophysical parameters and ocean surface winds. Since 2018, she has been with the Planetary Radar and Radio Science Group, Jet Propulsion Laboratory (JPL), Pasadena, CA, USA. She has engaged with Goldstone Solar System Radar (GSSR) signal processing activities. She continues to explore applications of GNSS-R in Earth science, while she expands her knowledge to planetary science to sense the properties of solar system bodies.

Dr. Rodriguez-Alvarez has made relevant accomplishments on wetlands, polar sea ice, and floods for which she has received the NASA Early Career Public Achievement Award in 2019, while at JPL.

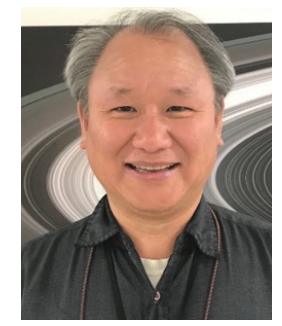

Joseph S. Jao is a Software Engineer with the Planetary Radar and Radio Science Group, Jet Propulsion Laboratory (JPL), Pasadena, CA, USA. $\mathrm{He}$ is developing remote control and system monitoring capabilities for radar operations. The goal is to allow U.S. investigators to have appropriate command and control of the observations from approved institutions using the Internet as the communications channel.

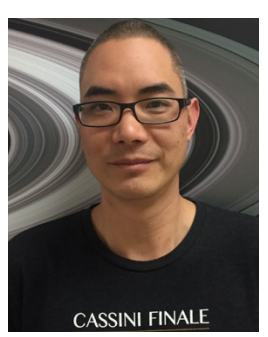

Clement G. Lee received the B.S. degree in electrical engineering from UCLA, Los Angeles, CA, USA, in 1999, and the M.S. degree in electrical engineering from USC, Los Angeles, CA, in 2003.

$\mathrm{He}$ has over 17 years of experience on planetary radar and radio science operations. He is a Senior Member of the Planetary Radar and Radio Science Group, NASA's Jet Propulsion Laboratory, Pasadena, CA, USA. He is the Goldstone Solar System Radar (GSSR) System Engineer. He has wide expertise on signal processing in radar and communications systems, field-programmable gate arrays (FPGAs), embedded software systems, and closed-loop and open-loop receivers. Over the years, he has supported numerous GSSR asteroid tracks and radio science operations, including Cassini finale year, Insight EDL, Mars Express, and Parker Solar Probe.

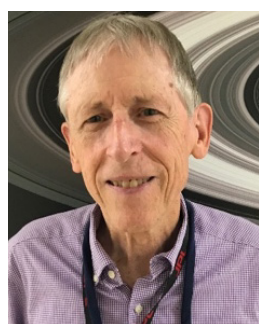

Martin A. Slade (Member, IEEE) received the B.S. and M.S. degrees in physics and the Ph.D. degree in earth, atmospheric, and planetary science from the Massachusetts Institute of Technology, Cambridge, MA, USA, in 1964, 1967, and 1971, respectively.

$\mathrm{He}$ is a member of the Planetary Radar and Radio Science Group, Jet Propulsion Laboratory (JPL), California Institute of Technology, Pasadena, CA, USA. His scientific interests have ranged from testing of gravitational theories that generalize "metric" theories (such as general relativity) to very long baseline observations of the structure of quasars and active galactic nuclei. At present, determinations of time variations in the spin vector and rotation period for both Mercury and Venus are ongoing collaborative research areas in radar astronomy. Other scientific interests are radar mapping of the topography of the lunar poles and the surface of Mercury. The determination by radar of the shapes, orbits, and physical characterization for near-Earth asteroids and comets is an active area of collaboration with scientists at JP Land elsewhere.

Dr. Slade is a member of the American Geophysical Union, the American Astronomical Society (Division for Planetary Sciences and Division on Dynamical Astronomy), the International Astronomical Union, and the Committee on Space Research (COSPAR)

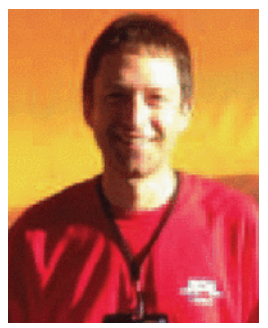

Joseph Lazio received the Ph.D. degree from Cornell University, Ithaca, NY, USA, in 1997.

$\mathrm{He}$ was a U.S. National Research Council Research Associate with the U.S. Naval Research Laboratory, Washington, DC, USA, where he was a Radio Astronomer. He was the Deputy Principal Investigator for the proposed Dark Ages Radio Explorer (DARE). He was a Project Scientist of the Square Kilometer Array (SKA), the Deputy Director of the Lunar University Network for Astrophysics Research (LUNAR), and a Project Scientist for the U.S. Virtual Astronomical Observatory. He is also a Directorate Scientist of the Interplanetary Network Directorate with the Jet Propulsion Laboratory, California Institute of Technology, Pasadena, CA, USA. The Interplanetary Network Directorate manages the Deep Space Network for NASA's Space Communications and Navigation (SCaN) Program. He also observes routinely with the world's premier ground-based radio telescopes, including the Jansky Very Large Array, the Very Long Baseline Array, the Green Bank Telescope, the Giant Metrewave Radio Telescope, and the Australia Telescope Compact Array. 


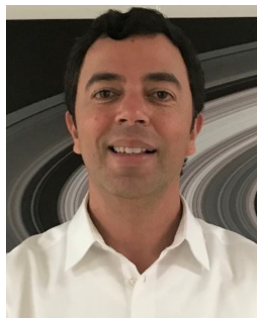

Kamal Oudrhiri is the Deputy Section Manager of the Communications Architectures and Research Section, Jet Propulsion Laboratory (JPL), Pasadena, CA, USA. He has over 17 years of experience in Technical Management, Radio Science, End-to-End Systems Engineering, Cognizant Engineering, and Deep Space Projects operations, specializing in planetary radar and radio science. He has managed multidisciplinary teams through the development and delivery of the Cold Atom Laboratory (CAL), and Ultra Stable Oscillators for Mars Reconnaissance Orbiter (MRO) and Gravity Recovery and Interior Laboratory (GRAIL) projects within cost and schedule. He is the CAL Mission Manager. His research focuses on planetary atmospheric characterization, gravity science, and bistatic radar. He has served in key roles on multiple NASA missions: The Mars Exploration Rovers (MER), the Cassini mission to Saturn, the GRAIL lunar mission, the Mars Science Laboratory (MSL), the Juno mission to Jupiter, and the New Horizons mission to Pluto.

Mr. Oudrhiri received three NASA's JPL Mariner and Voyager Honor Awards and multiple NASA Group Achievement Awards. He was a recipient of the People Leadership Award, which recognizes exceptional people leadership skills critical to the ongoing success of NASA's exploration missions.

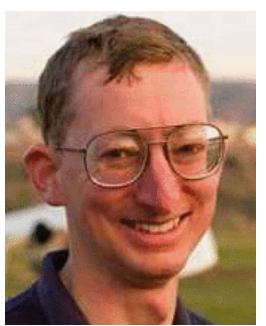

Kenneth S. Andrews received the B.S. degree in applied physics from the California Institute of Technology, Pasadena, CA, USA, in 1990, and the $\mathrm{Ph} . \mathrm{D}$. degree in electrical engineering from Cornell University, Ithaca, NY, USA, in 1999.

He has worked extensively in error correcting code design and implementation, particularly for low-density parity check and turbo codes. His work includes free-space optical communications and solar system radar.

Dr. Andrews is a member of the Communications Architectures and Research Section, Jet Propulsion Laboratory, California Institute of Technology.

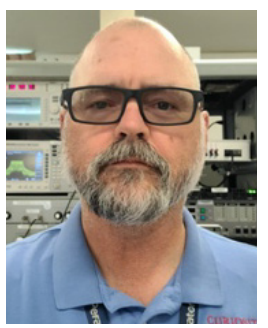

Lawrence G. Snedeker is the Supervisor of the Advanced Development/Unique Services at the Goldstone Seep Space Communications Complex. He is with Peraton Inc., Monrovia, CA, USA, and Jet Propulsion Laboratory, California Institute of Technology, Pasadena, CA, USA.

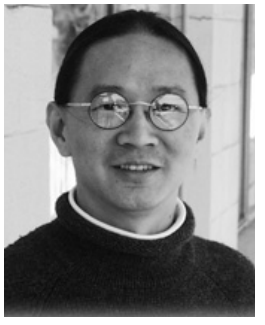

Ronglin R. Liou was born in Taiwan. He received the Ph.D. degree in electrophysics from the University of Southern California (USC), Los Angeles, CA, USA, in 1994.

$\mathrm{He}$ continued the postdoctoral research at USC, in gyrotron experiments involving rectangular and cylindrically tapered cavities. During this time, he also led experimental efforts on a novel microwave source generated by a laser-induced ionization front, a scheme now known as DARC (dc-to-ac radiation converter). In 1996, he joined Hughes Aircraft Company, Electron Dynamics Division (EDD), Torrance, CA, USA, first in developing linear traveling wave tube (TWT) for terrestrial applications and then as the technical lead for linearized traveling wave tube amplifiers (LTWTA) for satellite applications. He joined JPL in 2005 and has several flight projects, such as Aquarius, Mars Scientific Laboratory (Curiosity), Mars 2020 (Perseverance), and Surface Water and Ocean Topography (SWOT). Since 2009 and at Deep Space Network (DSN), his main focus has been on the development and deployment of high-power klystrons and transmitters.

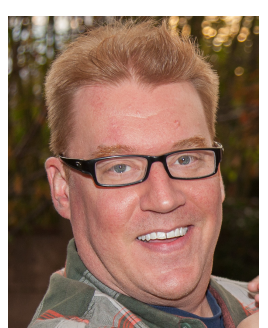

Kevin A. Stanchfield has been the Software Systems Engineer for Goldstone Solar System Radar (GSSR) with the Telecommunications Division, Jet Propulsion Laboratory, California Institute of Technology, Pasadena, CA, USA, since 2000, where he is an Information Technology (IT) Security Specialist. 